G300

PERINATAL ARTERIAL ISCHAEMIC STROKE - A MULTILEVEL COLLABORATIVE RETROSPECTIVE REGIONAL STUDY

${ }^{1} S$ Foulkes, ${ }^{2} \mathrm{MC}$ Wong, ${ }^{2} \mathrm{~K}$ Gallagher, ${ }^{3} \mathrm{~T}$ Austin, ${ }^{2} \mathrm{M}$ Chitre. 'School of Clinical Medicine, University of Cambridge, Cambridge, UK; ${ }^{2}$ Department of Paediatric Neurology, Cambridge University Hospitals, Cambridge, UK; ${ }^{3}$ Rosie Maternity Hospital, Cambridge University Hospitals, Cambridge, UK

\subsection{6/archdischild-2018-rcpch.292}

Aims Perinatal arterial ischaemic stroke (PAIS) presents in the neonatal period or later ('presumed'cases) and is a leading cause of hemiplegic cerebral palsy. Accurate estimation of the incidence and disease burden is challenging and studies of presumed cases are few. Our region covers 5 counties and has a population of approximately 5 million. We aim to review the regional clinical and radiological characteristics of children with PAIS through collaboration between one tertiary neonatal centre and an epilepsy network. The preliminary data is presented.

Methods Term neonates diagnosed with acute PAIS presenting to the tertiary neonatal unit in 2014 and 2015 were identified through electronic patient record (Badgernet) and a local database. Presumed PAIS cases presenting to the tertiary paediatric neurology unit between 2011-2015 were identified though clinical coding. Members of an established regional epilepsy network consisting of community, secondary, and tertiary paediatric clinicians were invited to identify local presumed PAIS cases over the same time period. Retrospective data was collected through medical records. Results have been received from 6 units to date and data collection is ongoing.

Results

- Neonatal cases all presented with seizures. While the majority $(78 \%)$ of presumed cases presented with pathological handedness, there is a male $(60 \%)$ and left sided infarct $(62.5 \%)$ predominance.

- $21 \%$ of infants with presumed PAIS cases had neonatal symptoms. These included changes in muscle tone (60\%), level of alertness (20\%) and feeding problems (40\%).

- Amongst all PAIS cases there was a significant burden of language (31\%), visual (21\%), behavioural (10\%) and learning impairment $(26 \%)$ in addition to the recognised movement difficulties and seizures.

Conclusion The clinical characteristics of PAIS in our region are similar to the published literature. Clinicians should have a high index of suspicion in neonatal cases of unexplained changes in muscle tone, respiratory or feeding difficulties which may lead to earlier diagnosis and intervention. Ongoing studies using a network approach would allow presentation of the wider spectrum of PAIS, producing more representative clinical data.

\section{G301 CEREBRAL VENOUS THROMBOSIS IN CHILDHOOD TUBERCULOUS MENINGITIS}

${ }^{1} S$ Dhawan, ${ }^{1} \mathrm{~N}$ Sankhyan, ${ }^{1} \mathrm{~J}$ Sahu, ${ }^{2} \mathrm{~S}$ Vyas, ${ }^{3} \mathrm{P}$ Singhi. ${ }^{1}$ Pediatrics, Postgraduate Institute of Medical Education and Research, Chandigarh, India; ${ }^{2}$ Radiodiagnosis, Postgraduate Institute of Medical Education and Research, Chandigarh, India; ${ }^{3}$ Pediatric Neurology and Neurodevelopment, Medanta, The Medicity, Gurgaon, India

\subsection{6/archdischild-2018-rcpch.293}

Background Few case reports have described cerebral venous sinous thrombosis (CVT) in children with tuberculosis and tubercular meningitis
Aims and objectives To determine incidence of CVT in children with tubercular meningitis

Methods This was a single centre prospective cohort of children diagnosed with TBM. The neuroimaging of children with TBM were prospectively evaluated for CVT. Children with CVT were treated with anti-tubercular drugs, steroids and anti-coagulation. Outcome was assessed using Paediatric Cerebral Performance Category Scale

Results 255 children with tubercular meningitis were evaluated out of which twelve children (4.7\%) had CVT. Median Age at diagnosis was 24 month (range 12-120 months). Median duration of symptoms was 59.5 days (IQR 30.5-77.5 days, range 18-159 days). Only one child had CVT at admission while ten children (83\%) were suspected to have CVST on CT scan subsequent to drainage of hydrocephalous. CVT was symptomatic in 33\% of children. Eleven children had involvement of superior sagittal sinus, six had involvement of transverse sinus, four had sigmoid sinus while one child also had affection of straight sinus. Two children succumbed during treatment. Protein $\mathrm{C}$ was deficient in one child while protein $\mathrm{S}$ was deficient in 3 children. Median Duration of follow up was 8 months. 2 children had normal functioning, 1 has severe disability, 5 are in vegetative state, one has moderate disability and cognitive impairment and 3 children have died

Conclusion CVT in TBM is not common and needs to be considered in any child who fails to have improvement in sensorium after CSF drainage procedure

\section{G302 COMPARISON OF 4-WEEKS VERSUS 12-WEEKS ANTI- CONVULSANT THERAPY FOR ACUTE SYMPTOMATIC SEIZURES IN CHILDREN WITH ACUTE ENCEPHALITIS SYNDROME- AN OPEN-LABEL, RANDOMISED CONTROLLED TRIAL}

'S Dhawan, 'J Sahu, ² Singhi, 'N Sankhyan, 'J Murlidharan. 'Pediatrics, Postgraduate Institute of Medical Education and Research, Chandigarh, India; ${ }^{2}$ Pediatrics, Medanta, The Medicity, Gurgaon, India

\subsection{6/archdischild-2018-rcpch.294}

Background There exists poor evidence-base and conflicting literature regarding optimum duration of anti-epileptic drugs for acute symptomatic seizures in central nervous system infections. A duration of one to three-months of anti-epileptic drugs has been suggested for central nervous system infections with parenchymal involvement. The study was designed to compare the effectiveness of 4 weeks versus 12 weeks anticonvulsant treatment in preventing seizure recurrences over a six-month period.

Methods Children aged 3 months to 12 years having Acute Encephalitis Syndrome with acute symptomatic seizures receiving single anti-epileptic drug at 4 weeks of illness and without seizure recurrence from day 7 day 28 of illness were included in this comparative, parallel group assignment, open label, randomised control study. The exclusion criteria were included children with chronic meningitis, brain abscess, intracranial space occupying lesion, prior history of seizures, prior focal neurological deficit or any developmental delay, children suffering from HIV, chronic liver/kidney disease, acute hepatic encephalopathy. Children on $\geq 2$ anti-epileptic drugs, severely affected children [Paediatric Cerebral Performance Category Scale (PCPC) Score and Paediatric Overall Performance Category Scale with category score of 5] were also excluded. They were randomly allocated to receive anti-epileptic drugs either 
for 4 weeks or 12 weeks. Children were followed up at 1, 3, and 6 months of illness. The primary outcome was proportion of children developing seizure recurrence over 6 months follow up. The secondary outcome was to study factor(s) associated with seizure recurrence.

Results Out of 232 children with Acute Encephalitis Syndrome, 60 children were found to be eligible for randomization in two groups. Baseline demographics were comparable (except duration of illness) between the groups. None of the children developed any seizure recurrences in the follow up period. Although, 8 children had neurological deficits and 9 children had EEG abnormality, seizure recurrences were not seen in any of these children.

Conclusions The present study suggests that a shorter duration (4 weeks) of anti-epileptic drug therapy is comparable with 12 weeks anti-epileptic drugs for preventing seizure recurrences over a six-month follow-up period in this cohort of children with Acute Encephalitis Syndrome.

The trial was registered with Clinical Trial Registry of India (CTRI/2017/06/008783) and Clinicaltrial.gov (NCT03181945).

\section{G303 ACUTE CLINICAL EMERGENCIES ON THE PAEDIATRIC NEUROSCIENCES WARD: CAN WE IMPROVE PREDICTION AND REDUCE RISK?}

${ }^{1}$ MR Eyre, ${ }^{2}$ L Andre, ${ }^{1}$ R Robinson. ${ }^{1}$ Paediatric Neurology Department, Great Ormond Street Hospital for Children NHS Foundation Trust, London, UK; ${ }^{2}$ Resuscitation Department, Great Ormond Street Hospital for Children NHS Foundation Trust, London, UK

\subsection{6/archdischild-2018-rcpch.295}

Background Paediatric inpatients with complex neurological problems can rapidly deteriorate and arrest on the ward. Various early warning risk-scoring systems are used, but none apply specifically to this patient cohort. The aim of this study was to identify modifiable risk factors and optimise patient safety.

Methods Our tertiary neurosciences unit comprises 24 acute beds (8 HDU) serving children with complex neurological, neurosurgical and craniofacial disorders. All calls to the clinical emergency team (CET) were prospectively audited.

Results Over 10.2 years the CET responded to 128 calls in 98 children (median age 2.8 y, range 2 d-19 y, 52\% female). Diagnoses included epilepsy (37\%), hydrocephalus (31\%), CNS tumour (14\%), craniofacial disorders (8\%) and epilepsy surgery (4\%). $19 \%$ of events followed recent surgery or general anaesthetic. Most recent Children's Early Warning Score (CEWS) was median 1 (IQR 0-3) at median 56 min pre-event (IQR 29-110).

Events included respiratory arrest (88\%) and cardiac arrest (4.7\%). Utstein-style categorization was used to classify the primary cause: $72 \%$ neurological, including seizure-related apnoea in $41 \%$ and raised intracranial pressure in $25 \%$ (hydrocephalus 16\%, acute haemorrhage 5.5\%); 22\% respiratory, including central or obstructive apnoea in $9.4 \%$, benzodiazepine-related apnoea in $6.3 \%$, and blocked tracheostomy in $4.7 \% ; 3.1 \%$ circulatory (septic shock, hypovolaemia). 33\% of events were regarded as potentially preventable by the attending CET.

In addition to basic supportive care, interventions on the ward included endotracheal intubation in 16\%, mannitol/ hypertonic saline in $9.4 \%$ and adrenaline in $2.3 \%$. No shockable rhythms were identified. One death occurred during resuscitation and $33 \%$ of survivors were transferred to ICU; all were alive at 24 hours. In 11 consecutive patients prospectively followed up, all survived to discharge at median 11 days (range 3-93) post-event; 86\% were alive at 1 year.

Conclusions Respiratory arrests secondary to epileptic seizure, raised intracranial pressure, central or obstructive apnoea and benzodiazepine administration are potentially predictable. CEWS had poor sensitivity for predicting imminent deterioration in paediatric neurosciences patients. Analysis of pre-event clinical observations in cases and controls will enable development of a deterioration-prediction model specific to this patient cohort.

\section{G304 DELPHI CONSENSUS PROCESS FOR THE UK GUIDELINES FOR MANAGEMENT AND SURVEILLANCE OF TUBEROUS SCLEROSIS COMPLEX}

${ }^{1} \mathrm{~S}$ Amin, ${ }^{2} \mathrm{C}$ Kingswood, ${ }^{3} \mathrm{~F}$ O'Callaghan. 'University Hospitals Bristol, Bristol, UK; ${ }^{2}$ Royal Brighton Hospital, Brighton, UK; ${ }^{3}$ University College London, London, UK

10.1136/archdischild-2018-rcpch.296

Background Tuberous Sclerosis Complex (TSC) is a multi-systemic genetic disease. The severity of TSC can vary among affected individuals. Complications of TSC can be life threatening, with significant impact on patients' quality of life. Management may vary dependent on treating physician, local and national policies and funding. There are no current UK guidelines. We conducted a Delphi consensus process to gain consensus on the management of patients with TSC in the UK.

Methods We invited 86 clinicians and researchers to complete an online survey in two rounds. All the people surveyed were based in the UK. Clinicians were identified through the regional TSC clinics, and researchers were identified through publications. In round one, 55 questions were asked. They were related to surveillance and management recommendations for those newly diagnosed, suspected, or already diagnosed with TSC. In round two, 18 questions were asked to obtain consensus on the outstanding points that had been contentious in round one or needed clarification.

Results 51 (60\%) responded to the survey. Three rounds were required to achieve consensus. The responders were neurologists, nephrologists, psychiatrists, psychologists, oncologists, general paediatricians, dermatologists, urologists, radiologists, geneticists, neurosurgeons, pulmonologists and neurodisability clinicians. A priori consensus was defined as $70 \%$ agreement among participants. The Delphi process is now complete and the consensus management recommendations will be presented at conference.

Conclusions This new UK guideline for the management and surveillance of TSC patients provides a realistic, cost effective, an evidence-based approach for best clinical care delivered for individuals with tuberous sclerosis complex in the UK.

\section{G305 HOW MUCH VARIATION IN PAEDIATRIC EPILEPSY ADMISSION RATES IN ENGLAND CAN BE EXPLAINED BY VARIATION IN UNIT-LEVEL PERFORMANCE IN THE NATIONAL CLINICAL AUDIT (EPILEPSY12)}

${ }^{1} S$ Arora, ${ }^{1} \mathrm{C}$ Sherlaw-Johnson, ${ }^{2} \mathrm{CR}$ Cheung, ${ }^{3} \mathrm{DS}$ Hargreaves. ${ }^{1}$ Research Department, The Nuffield Trust, London, UK; ${ }^{2}$ Department of General Paediatrics, Evelina London Children's Hospital, London, UK; ${ }^{3}$ Population, Policy and Practice Programme, UCL Great Ormond Street Institute of Child Health, London, UK

10.1136/archdischild-2018-rcpch.297 